\title{
DIFFERENTIAL AND INTEGRAL INEQUALITIES
}

\section{RAYMOND M. REDHEFFER}

This note presents new proofs for some important inequalities [1]. The assumptions on positivity or monotony of the various functions are weaker than those in [1] or in the original references (see [1]) and yet the method seems astonishingly elementary. We set $u^{\prime}$ $=d u / d t$, and $t \geqq 0$; the reversal of inequalities for $t<0$ can be read off from the proofs. Since continuous functions are dense in the integral norm, we suppose all functions continuous.

Let $T u=u^{\prime}-a(t) b(u)$, where $b>0$. Then

$$
T u \leqq 0 \leqq T v \text { and } u(0) \leqq v(0) \Rightarrow u \leqq v .
$$

For proof define $\tilde{u}=B(u)$ and $\tilde{v}=B(v)$, where $B(y)=\int_{\delta}^{\nu}[b(s)]^{-1} d s$. Then

$$
b(u)\left[\tilde{u}^{\prime}-a(t)\right] \leqq 0 \leqq b(v)\left[\tilde{v}^{\prime}-a(t)\right],
$$

therefore $\tilde{u}^{\prime} \leqq a(t) \leqq \tilde{v}^{\prime}$, and therefore $\tilde{u}-\tilde{v}$ is nonincreasing. But $\tilde{u}(0) \leqq \tilde{v}(0)$. Hence $\tilde{u} \leqq \tilde{v}$, and consequently $u \leqq v$. The proof shows that the differential inequality is needed only at points where $u>v$.

The equation $T v=0, v(0)=\delta$ can be solved by inspection, to give:

(2) $T u \leqq 0$ and $u(0) \leqq \delta \Rightarrow u \leqq B^{-1}[A(t)]$, where $A(t)=\int_{0}^{t} a(s) d s$.

In this paragraph only, let $u$ and $v$ be vectors, with any convenient norm, and let $\widetilde{T} u=u^{\prime}-f(t, u)$, where $f$ is a vector. Suppose

$$
\|f(t, u)-f(t, v)\| \leqq a(t) b(w) \quad \text { where } \quad w=\|u-v\| .
$$

If $\tilde{T} u=\tilde{T} v$ and $\|u-v\|=\delta$ for $t=0$, then the fact that $\left|\|w\|^{\prime}\right| \leqq\left\|w^{\prime}\right\|$ gives

$$
p w^{\prime} \leqq\left\|u^{\prime}-v^{\prime}\right\| \leqq a(t) b(w), \quad p=+ \text { or } p=-
$$

Without loss of generality take $b$ even, so $b(p w)=b(w)$. Let $v=v^{p}$ satisfy $(p v)^{\prime}=a(t) b(p v), v(0)=\delta$. Then $T(p w) \leqq 0=T(p v), \quad p w(0)$ $=p v(0)$. Hence $p w \leqq p v^{p}$, which is to say, $v^{-} \leqq w \leqq v^{+}$. Since $v^{p}$ satisfies $v^{\prime}=p a(t) b(v)$, we use $p A$ in (2) and get:

$$
B^{-1}[-A(t)] \leqq\|u-v\| \leqq B^{-1}[A(t)] .
$$

This sharpens the theorems of Bihari and Langenhop.

Received by the editors April 26, 1963. 
If $a \geqq 0$ we can allow $b \geqq 0$ instead of $b>0$ : Replace $b$ by $b+h$, where $h$ is a positive constant. For the corresponding operator $T_{h}$ we have $T_{h} u \leqq T u \leqq 0$, hence $u \leqq v_{h}$. The resulting inequality for $h \rightarrow 0$ depends on the convergence or divergence of the integral defining $B$ at the zeros of $b$. Since equation (2) for $v_{h}$ can be written

$$
T u \leqq 0 \text { and } u(0) \leqq \delta \Rightarrow u \leqq y, \text { where } \int_{\delta}^{y} \frac{d s}{b(s)+h} \geqq \int_{0}^{t} a(s) d s,
$$

the behavior as $h \rightarrow 0$ is analyzed with ease.

When not only $a \geqq 0$ but $b$ is monotone nondecreasing as a function of its argument, $u$, we can analyze the integral inequality

$$
w \leqq \delta+\int_{0}^{t} a(s) b[w(s)] d s .
$$

If the right side is called $u$ then $u^{\prime}=a(t) b(w) \leqq a(t) b(u)$, so that $T u \leqq 0, u(0)=\delta$. Therefore the estimate (2) holds for $u$, and $a$ fortiori for $w$. This sharpens another theorem of Bihari. The choice $\delta=a=1$, $b(w)=4(w+1)^{-1}, w=t^{2}, t=2$, shows that the monotony of $b$ cannot be dropped here, although it was superfluous in (1).

\section{REFERENCE}

1. E. F. Beckenbach and Richard Bellman, Inequalities, pp. 133-136, Springer, Berlin, 1961.

University of California, Los Angeles 